\title{
Anti-malarial activity of a polyherbal product (Nefang) during early and established Plasmodium infection in rodent models
}

Protus Arrey Tarkang ${ }^{1,2^{*}}$, Faith A Okalebo ${ }^{2}$, Lawrence S Ayong ${ }^{3,4}$, Gabriel A Agbor ${ }^{1}$ and Anastasia N Guantai ${ }^{2}$

\begin{abstract}
Background: The emerging resistance of Plasmodium species to currently available anti-malarials remains a public health concern, hence the need for new effective, safe and affordable drugs. Natural products remain a reliable source of drugs. Nefang is a polyherbal anti-malarial of the Cameroonian folklore medicine with demonstrated in vitro antiplasmodial and antioxidant activities. It is composed of Mangifera indica (bark and leaf), Psidium guajava, Carica papaya, Cymbopogon citratus, Citrus sinensis, Ocimum gratissimum (leaves). This study aimed at investigating the suppressive, prophylactic and curative activities of Nefang in Plasmodium infected rodent models.

Methods: Systemic acute oral toxicity of Nefang aqueous and ethanol extracts was assessed in mice up to a dose of 5,000 mg kg-1 body weight. BALB/c mice and Wistar rats were inoculated with Plasmodium chabaudi chabaudi and Plasmodium berghei, respectively, and treated with Nefang, the Mangifera indica bark/Psidium guajava combination and a Psidium guajava leaf aqueous extracts (75, 150, 300 and $600 \mathrm{mgkg}^{-1} \mathrm{bwt}$ ). Their schizonticidal activity was then evaluated using the Peter's 4-day suppressive test). The prophylactic and curative (Rane's Test) activity of Nefang was also evaluated by determining the parasitaemia, survival time, body weight and temperature in pre-treated rodents.

Results: Acute oral toxicity of the extract did not cause any observed adverse effects. Percent suppressions of parasitaemia at $600 \mathrm{mgkg}^{-1}$ bwt were as follows (P. berghei/P. chabaudi): Nefang - 82.9/86.3, Mangifera indica bark/Psidium guajava leaf combination extract - 79.5/81.2 and Psidium guajava leaf - 58.9/67.4. Nefang exhibited a prophylactic activity of $79.5 \%$ and its chemotherapeutic effects ranged from $61.2-86.1 \%$ with maximum effect observed at the highest experimental dose.
\end{abstract}

Conclusion: These results indicate that Nefang has excellent in vivo anti-malarial activities against $P$. berghei and $P$. chabaudi, upholding earlier in vitro antiplasmodial activities against multi-drug resistant $P$. falciparum parasites as well as its traditional use. Hence, Nefang represents a promising source of new anti-malarial agents.

Keywords: Medicinal Plants, Nefang, Acute toxicity, Malaria, In vivo antiplasmodial activity, Suppressive activity, Prophylactic activity, Curative activity, Combination phytotherapy

\footnotetext{
* Correspondence: ptarkang@yahoo.co.uk

${ }^{1}$ Centre for Research on Medicinal Plants and Traditional Medicine, Institute of Medical Research and Medicinal Plants Studies (IMPM), P. O. Box 6163,

Yaoundé, Cameroon

${ }^{2}$ Department of Pharmacology and Pharmacognosy, University of Nairobi,

P. O. Box 19676-00202, Nairobi, Kenya

Full list of author information is available at the end of the article
} 


\section{Background}

Despite substantial efforts to control malaria in the last few decades, it remains one of the most prevalent infectious diseases globally. The emerging resistance of Plasmodium species to currently available drugs remains a public health concern [1]. Forty percent of the world's population is exposed to malaria and there is a constant need for new antimalarials. Historically, plants have had a remarkable role in therapeutics and were the principal source of drugs for many centuries. Quinine, isolated in 1820 from Cinchona species (Rubiaceae), is an illustrative example. Drugs in current use for malaria chemotherapy include artemisinin, from Artemisia annua (Asteraceae) of Chinese origin, and its semi-synthetic derivatives, artemether, artesunate and arteether [2]. A recently introduced plant-derived antimalarial drug is atovaquone, a synthetic naphthoquinone based on lapachol. Lapachol, a prenylnaphtoquinone, was first isolated from Tabebuia impetiginosa, a South American member of the Bignoniaceae family [3]. Artemisinin-based combination therapy (ACT) is currently the most effective chemotherapy against Plasmodium falciparum malaria and the emergence of resistance would be a public health disaster in malaria endemic areas. Therefore, plants do not only provide valuable clues for finding new drugs, but may help to shift the drug discovery paradigm from finding new molecules to combining existing agents $[4,5]$.

The modern pharmaceutical industry was born from botanical medicine, but standardized synthetic combinatorial chemistry in drug discovery and high throughput screening (HTS) of potential drug targets have disconnected the historical link between plants and medicines. However, this has been rekindled by the small output of modern anti-malarial pharmaceutical research and development, which has stimulated new interest in the potential of natural compounds [6]. Hence natural products continue to provide new starting points in drug discovery.

There is a school of thought that biologically-derived secondary metabolites and synthetic compounds derived from them perform better as drugs than randomly synthesized compounds. Drug-derived parent molecules were present in primitive life forms and therefore co-evolved to interact with one another, thus granting direct ecological benefit to the producing organism, whether in competition for resources, avoiding predation or combating pathogens [7]. This co-evolution between different plants and/or metabolites within the same plant brings about synergy or potentiation which has been proven to achieve favourable results, such as enhanced efficacy, decreased dosage at equal or increased level of target inhibition, reduced or delayed development of drug resistance and simultaneous reduction of toxic effects [8]. Based on this paradigm, drug combination in anti-malarial chemotherapy has been adopted and is widely used as a strategy to monitor and prevent resistance [9].
Nefang is a polyherbal product composed of the aqueous extracts of Mangifera indica (bark and leaf), Psidium guajava, Carica papaya, Cymbopogon citratus, Citrus sinensis and Ocimum gratissimum (leaves). It is frequently used for the treatment of malaria in the South West Region of Cameroon. Ethnopharmacological studies confirmed its formulation and folk use [10]. Studies have been conducted to evaluate the in vitro and in vivo antioxidant properties of this polyherbal [11], which may play a role in curbing oxidative stress related with malaria infection. The in vitro antiplasmodial activity of this product and solvent extracts of its constituents have also been evaluated, showing good activities and synergistic potentials of some constituent extracts [12].

This study aimed at evaluating the in vivo suppressive, prophylactic and curative activities of Nefang in mice and rats models. Results obtained showed that Nefang exhibited excellent in vivo anti-malarial activities in rodents, consistent with its previously observed anti-P. falciparum activities.

\section{Methods}

\section{Extraction of plant material}

Fresh parts of the constituent plants of Nefang: bark and leaves of Mangifera indica ( $M i B$ and $M i L$, respectively), and leaves of Psidium guajava (Pg), Carica papaya (Cp), Cymbopogon citratus (Cc), Citrus sinensis (Cs), Ocimum gratissimum $(O g)$ were harvested from their natural habitat in Cameroon between July and August 2011. Plant identification and voucher specimen referencing were done at the Institute of Medical Research and Medicinal Plants Studies (IMPM) herbarium in Yaoundé, Cameroon by a botanist. The freshly harvested plant parts were air dried and pulverized. Aqueous extraction was performed based on the traditional knowledge of preparation. Research evidence shows that ethanol extracts are as effective as the water extracts [13] and, therefore, ethanol extraction was also performed. Weighed quantities (1,000 g) of each plant part were exhaustively macerated in water $(2.4 \mathrm{~L})$ and ethanol $(2.0 \mathrm{~L})$ respectively for $4 \mathrm{~h}$. Each of the macerate was transferred into a conical percolator for $72 \mathrm{~h}$ and the extracts were filtered [14]. Each ethanol filtrate was first concentrated using a rotary evaporator. The filtrates were then concentrated in an air oven at $60^{\circ} \mathrm{C}$. The extracts were weighed and stored in labeled sealed plastic containers at $4^{\circ} \mathrm{C}$ until use to prevent decomposition.

\section{Experimental animals}

Swiss albino mice $(25-30 \mathrm{~g})$ were used for the acute toxicity testing while BALB/c mice $(20-25 \mathrm{~g})$ and Wistar rats $(160-180 \mathrm{~g})$ were used for in vivo antiplasmodial activities testing. All experimental animals were housed under standard environmental conditions of temperature 
at $22-24^{\circ} \mathrm{C}$ under a $12 \mathrm{~h}$ dark-light cycle, and allowed free access to drinking water and standard pellet diet.

Ethical approval for the study was obtained from Kenyatta National Hospital/University of Nairobi Ethics and Research committee, Nairobi-Kenya (KNH-ERC/A/324 5/12/12), Institute of Medical Research and Medicinal Plants Studies Institutional Review Board (076/82-62/ MINRESI/M000 - 01/06/12) and Institut Pasteur KoreaInstitutional Animal Care and Use Committee (IACUC No. IPK 12009 - 29/10/12).

\section{Acute (single dose) oral toxicity testing}

The acute oral toxicity of Nefang aqueous and ethanol extracts was evaluated according to the procedures outlined by the Organization for Economic Co-operation and Development [15]. Each crude extract was suspended in a vehicle (distilled water and corn oil for the aqueous and ethanol extracts respectively). Following a $4 \mathrm{~h}$ fasting period, mice were divided into groups of three. Extract doses were calculated in reference to mice body weight and each mouse was treated with a single oral dose of the extract.

The mice were dosed in a stepwise procedure using the fixed doses of 5, 50, 300, 1,200 and 2,000 $\mathrm{mgkg}^{-1}$ body weight (bwt) of the aqueous and ethanol extracts for each group of mice respectively. After each dose, the animals were observed for signs of toxicity for three hours. If there was no mortality or signs of toxicity at the highest dose, then the upper limit dose was used for the main test.

For the main test, a single high oral dose of 5,000 $\mathrm{mgkg}^{-1}$ bwt of each extract was administered to three male (Test 1) and three female (Test 2) mice in the treatment groups, whereas the control groups received the vehicle. Food was provided to the mice approximately an hour after treatment. The animals were observed $30 \mathrm{~min}$ after dosing, followed by hourly observation for $8 \mathrm{~h}$ and once a day for the next 13 days. Observations were systematically recorded for each animal. Surviving animals were weighed and visual observations for mortality, behavioural pattern, changes in physical appearance, injury, pain and signs of illness were conducted daily during the study period.

\section{Parasite infection of experimental animals}

The chloroquine sensitive strain of Plasmodium berghei (strain ANKA) was generously donated by the Institute of Primate Research (IPR), Nairobi, Kenya, while the Plasmodium chabaudi chabaudi was obtained from the Centre for Neglected Diseases Drug Discovery (CND3), Institut Pasteur Korea, as cryo-frozen stock of parasitized red blood cells (PRBCs). The parasites were prepared through two cycles of passage of the PRBCs in rats and mice. Donors with parasitaemia level of $20-30 \%$ were sacrificed and blood collected by cardiac puncture into heparinized tubes. The blood was then diluted with phosphate buffered saline (PBS) based on parasitaemia level of each donor and the RBC count of normal mice and rats, such that $1 \mathrm{~mL}$ blood contained $5 \times 10^{7}$ parasites. The experimental animals were each treated with $1 \times 10^{7}$ PRBCs by intraperitoneal (ip) injection [16].

\section{Test for suppressive activity (Peter's 4-day test)}

The aqueous extracts that were selected for this study were Nefang, Psidium guajava (Pg) (i.e. the most active constituent aqueous extract) and Mangifera indica bark/ Psidium guajava leaf $(\mathrm{MiB} / \mathrm{Pg})$ (i.e. the solvent extract combination that showed the most promising synergistic activity) [12]. The suppressive activities of these extracts were evaluated in early $P$. chabaudi and P. berghei infection in BALB/C mice and Wistar rats, respectively, using the method described by Knight and Peters [17]. Forty-five mice and forty-five rats were each randomly divided into fifteen groups of three each. On the first day (D0), the mice and rats were each infected with $10^{7} P$. chabaudi and $P$. berghei, respectively. Three hours later, the experimental groups of mice and rats were each treated orally with $10 \mathrm{mLkg}^{-1}$ bwt of the drug or extract as follows: Group 1 (negative/vehicle control) - PBS, Group 2 (positive control) - chloroquine $\left(10 \mathrm{mgkg}^{-1}\right)$, Group 3 (positive control) pyrimethamine (30 $\left.\mathrm{mgkg}^{-1}\right)$, Groups 4 to 7 - Nefang, Groups 8 to $11-P g$ and Groups 12 to $15-\mathrm{MiB} / \mathrm{Pg}$. The aqueous plant extracts were each administered orally at a dose of 75, 150, 300 and $600 \mathrm{mgkg}^{-1}$ respectively. Treatment was carried out for four consecutive days (D0 - D3). The body weight of each mouse were measured on the first day (D0) and on the fifth day (D4) using a sensitive digital analytical balance, while the body temperature was taken before infection and three hours after infection (D0) and then monitored daily to the fifth day (D4).

On the fifth day (D4), thin blood film was prepared from the tail blood of each experimental animal, fixed in methanol and stained with Giemsa to reveal parasitized erythrocytes. Parasitaemia was determined by light microscopy using a 100X objective lens and the following equation:

$$
\% \text { parasitaemia }=\frac{\text { No.of parasitized RBC }}{\text { Total no. of RBC counted }} \times 100
$$

Average percentage chemosuppression was calculated as

$$
100\left[\frac{A-B}{A}\right]
$$

where $A$ is the average percentage parasitaemia in the negative control group and $B$ is the average percentage parasitaemia in the test group. 


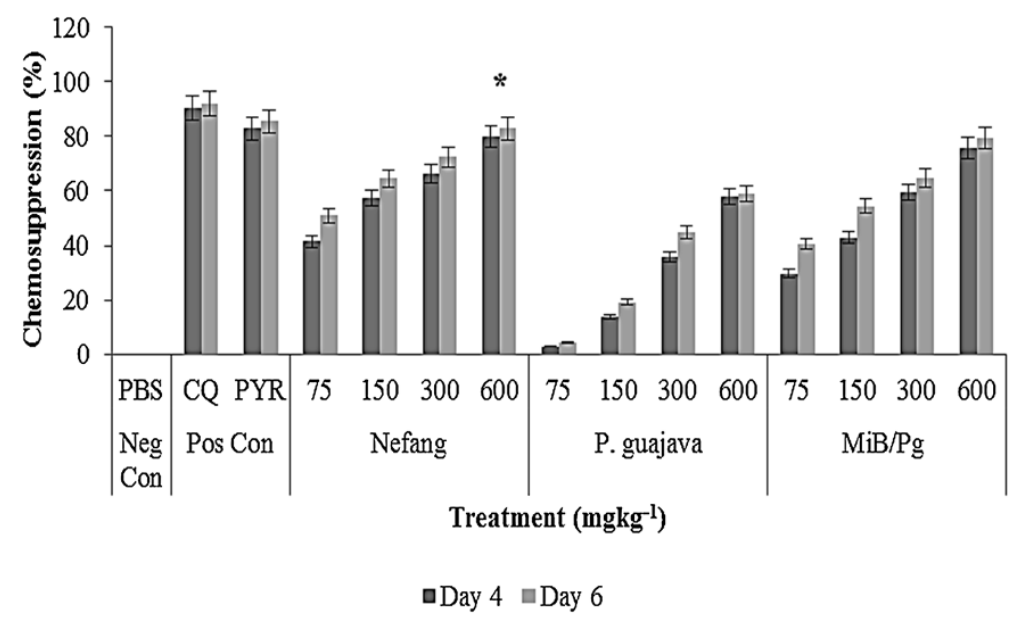

Figure 1 Suppressive activity of Nefang and active solvent extracts on $\boldsymbol{P}$. berghei infection in rats. Each bar represents the Mean \pm SD for each group of rats, $n=3$. Neg Con - negative control; Pos Con - positive control; CQ - chloroquine; PYR - pyrimethamine. *Best chemosuppression compared to control.

\section{Test for prophylactic activity}

The repository activity of Nefang was assessed using the method described by Peters [18]. The mice were randomly divided into seven groups of three BALB/c mice each. Group 1 (negative control) was treated with $10 \mathrm{mLkg}^{-1}$ of PBS, group 2 and 3 (positive controls) CQ $\left(10 \mathrm{mgkg}^{-1}\right)$ and pyrimethamine $\left(30 \mathrm{mg} / \mathrm{kg}^{-1}\right)$, respectively, group 4 to 7 (extract test groups) $-75,150$, 300 and $600 \mathrm{mgkg}^{-1}$ of Nefang, respectively. Administration of the extract and standard drugs continued for three consecutive days (D0 - D2). On the fourth day (D3), the mice were inoculated with $10^{7} \mathrm{P}$. berghei infected red blood cells and the level of parasitaemia was assessed by blood smear $72 \mathrm{~h}$ later.

\section{Test for curative activity (Rane's test)}

The schizontocidal activity of Nefang on established infection was evaluated using the method described by Ryley and Peters [19]. Twenty-one rats were infected with $P$. berghei $\left(10^{7}\right)$ PRBCs by ip injection on the first day (D0). Seventy-two hours later (D3), the rats were randomly divided into seven groups of three rats each. Four groups of the rats were treated orally with 75 , 150,300 and $600 \mathrm{mgkg}^{-1}$ bwt of Nefang respectively. The negative control group was treated with PBS while the two positive control groups were treated with CQ $\left(10 \mathrm{mgkg}^{-1}\right)$ and artesunate $\left(5 \mathrm{mgkg}^{-1}\right)$, respectively. Nefang and the standard drugs were each treated once daily for five days. Giemsa-stained thin smears were

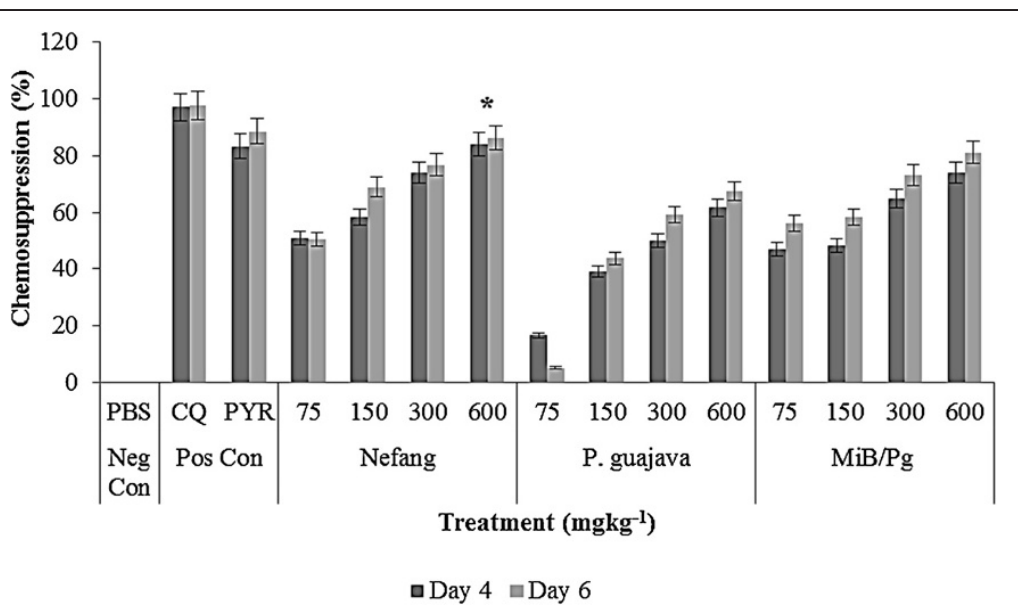

Figure 2 Suppressive activity of Nefang and active solvent extracts on $P$. chabaudi infection in mice. Each bar represents the Mean \pm SD for each group of rats, $\mathbf{n}=\mathbf{3}$. Neg Con - negative control; Pos Con - positive control; *Best chemosuppression compared to control. 
prepared from tail blood samples collected on each day of treatment to monitor parasitaemia level. The body weight and temperature were taken before infection (D0) and from the fourth day (D3) to the eighth day (D7) while the mean survival time (MST) of the rats in each treatment group was determined over a period of 29 days (D0 - D28) as follows;

$$
\text { MST }=\frac{\text { Number of days survived }}{\text { Total number of days }(29)} \times 100
$$

\section{Statistical analysis}

Data are expressed as mean \pm standard deviation (SD) of the mean. Data were analysed using SPSS Version 20.0. Statistical significance testing was done using the one-way analysis of variance (ANOVA) followed by Neuman-Keuls multiple comparison test. P-values of less than 0.05 were considered statistically significant.

\section{Results}

\section{Acute (single dose) oral toxicity testing}

There were no observed adverse effects at all oral dose levels $\left(5,50,300,2000 \mathrm{mgkg}^{-1}\right.$ bwt $)$ for all the aqueous

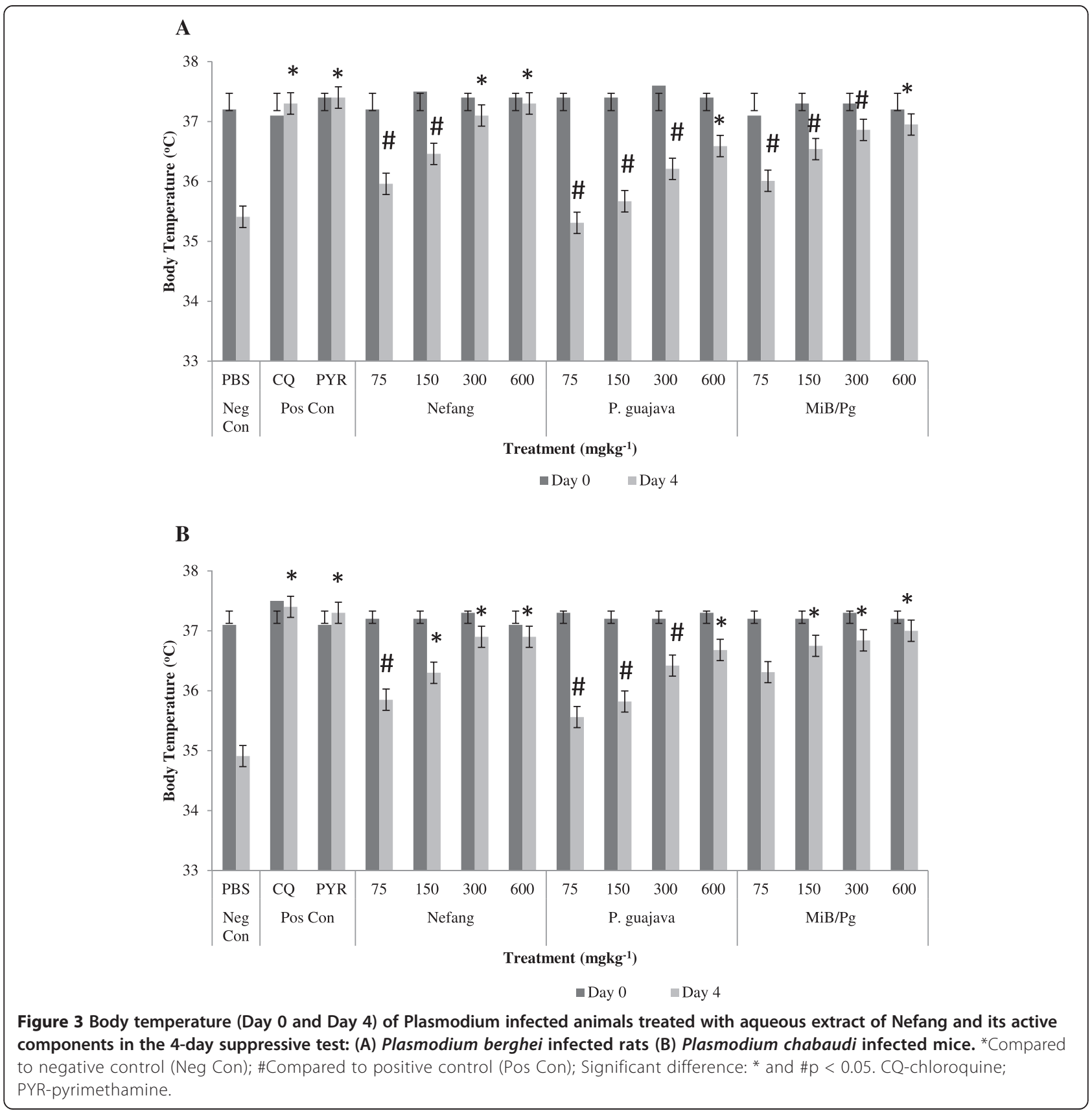


and ethanol extracts of Nefang and its constituents. All the mice survived. Similarly, oral administration of the aqueous and ethanol extracts of Nefang and its constituents at $5,000 \mathrm{mgkg}^{-1}$ bwt, had no toxic effects throughout the 14-day study period. None of the mice showed any signs of toxicity, such as changes on skin, eyes and mucus membranes, behavioural patterns, trembling, diarrhoea, falling of the fur, sleep or coma. No significant changes were observed in their body weights. The estimated maximum tolerable dose (MTD) was above $5,000 \mathrm{mgkg}^{-1}$ but for all extracts tested.
Evaluation of the suppressive activity (Peter's 4-Day Test) Nefang, Psidium guajava and $\mathrm{MiB} / \mathrm{Pg}$ all showed dosedependent chemosuppressive activities on parasitaemia. These effects were statistically significant $(\mathrm{p}<0.001)$ relative to the vehicle treated group. At all doses, the suppressive activity in rats (Figure 1) and mice (Figure 2) were comparable. On day 6, at the highest experimental dose of 600 $\mathrm{mgkg}^{-1}$, percentage suppression of parasitaemia were as follows (P. berghei/P. chabaudi): Nefang - 82.9/86.3, MiB/ $\mathrm{Pg}-79.5 / 81.2, \mathrm{Pg}-58.9 / 67.4$ as against CQ $\left(10 \mathrm{mgkg}^{-1}\right)-$ 92.1/97.7 and pyrimethamine $\left(30 \mathrm{mgkg}^{-1}\right)-85.5 / 88.5$. This

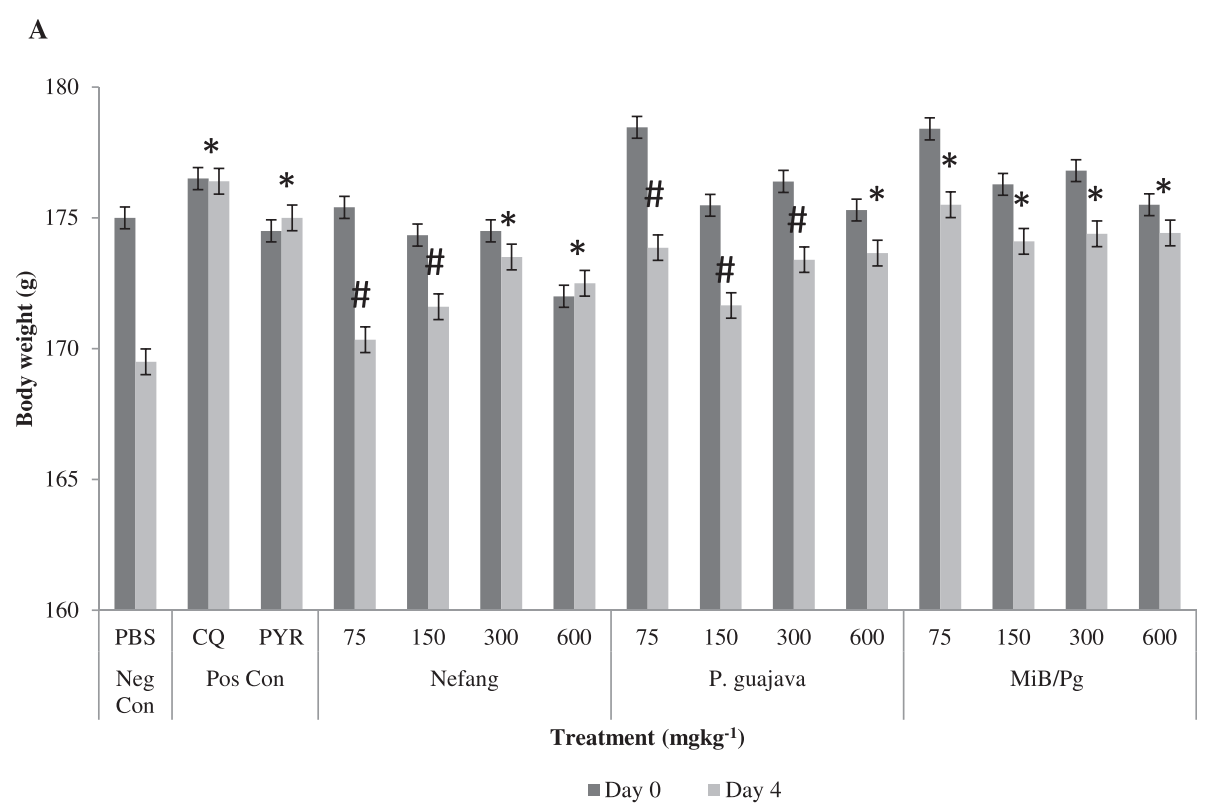

B

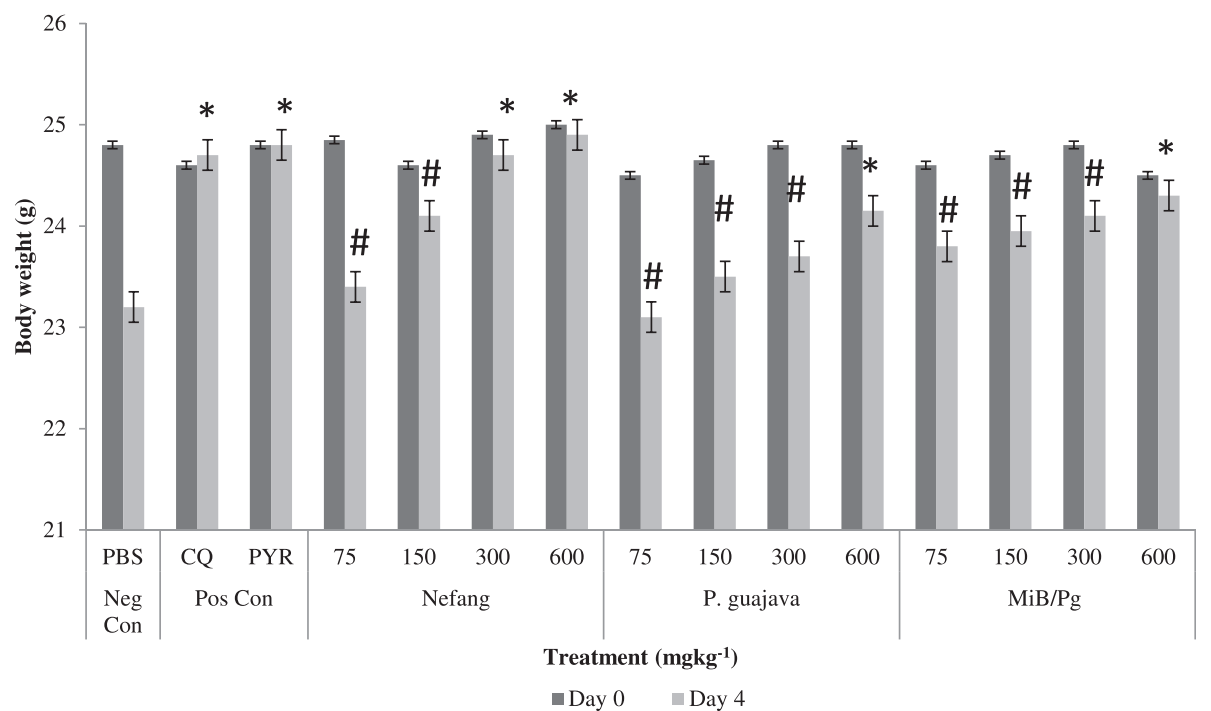

Figure 4 Body weight (Day 0 and Day 4) of Plasmodium infected animals treated with aqueous extract of Nefang and its active components in the 4-day suppressive test: (A) Plasmodium berghei infected rats (B) Plasmodium chabaudi infected mice. ${ }^{*}$ Compared to negative control (Neg Con); \#Compared to positive control (Pos Con); Significant difference: * and \#p < 0.05. CQ-chloroquine; PYR-pyrimethamine. 
shows that the in vivo activity of Nefang was comparable to that of pyrimethamine and better than for $M i B / P g$ and $P g$. It was significantly $(\mathrm{p}<0.05)$ lower than for $C Q$.

Nefang (300 and $\left.600 \mathrm{mgkg}^{-1} ; \mathrm{p}<0.01\right)$ and $\mathrm{MiB} / \mathrm{Pg}$ $\left(600 \mathrm{mgkg}^{-1} ; \mathrm{p}<0.05\right)$ caused significant attenuation of reduction in body temperature in both $P$. berghei infected rats and $P$. chabaudi infected mice in a dose-dependent manner (Figure 3). The effects of Nefang and $M i B / P g$ on the body temperature of experimental animals were comparable to those of the standard drugs CQ and PYR (Additional file 1). The extracts averted the loss of body weight associated with Plasmodium infection at same doses when compared to the control (Figure 4). No significant increases in weight were observed (see Additional file 2).

\section{Evaluation of the prophylactic activity}

Nefang aqueous extract showed a dose-dependent reduction of parasitaemia in experimental groups of mice. These reductions are statistically significant relative to the control. At the highest experimental dose of $600 \mathrm{mgkg}^{-1}$, Nefang demonstrated a high antiplasmodial activity of $79.5 \%$, which was very significant $(\mathrm{p}<0.001)$ when compared to the vehicle. Though slightly lower than that exhibited by CQ (86.9\%) at $10 \mathrm{mgkg}^{-1}$, it was comparable to that exhibited by PYR (78.4\%) at $30 \mathrm{mgkg}^{-1}$ (Figure 5).

\section{Evaluation of the curative activity (Rane's test)}

There was a dose-dependent reduction of parasitaemia in all Nefang experimental groups relative to the vehicle. These reductions were statistically significant $(\mathrm{p}<0.001)$ when compared to the vehicle. The dose-dependent chemosuppression exhibited by Nefang at a dose of $600 \mathrm{mgkg}^{-1}$ bwt was comparable to that of the standard drugs, CQ $\left(10 \mathrm{mgkg}^{-1}\right)$ and artesunate $\left(5 \mathrm{mgkg}^{-1}\right)$ (Figure 6; see Additional file 3). Nefang also demonstrated a significant $(\mathrm{p}<0.05)$ protective effect on the experimental rats as observed in the mean survival time of the animals, especially at the highest dose with all animals surviving through the experiment (Figure 7; see Additional file 4).

Reduction in body weight of infected animals was significantly $(\mathrm{p}<0.05)$ prevented by Nefang at doses of 300 and $600 \mathrm{mgkg}^{-1}$. This activity was comparable to that of the standard drugs, CQ and artesunate (Figure 8). At same doses, Nefang significantly $(\mathrm{p}<0.001)$ prevented the reduction in body temperature of infected animals and this was comparable to that of the standard drugs (Figure 9).

\section{Discussion}

The in vivo antiplasmodial activities of the aqueous extract of Nefang and its active components, $P g$ and $M i B / P g$, were investigated by evaluating the chemosuppression during early infection, while Nefang alone was evaluated during established infection using standard animal models. In vivo models are usually employed in anti-malarial studies because they take into account the possible prodrug effect and probable involvement of the immune system in eradication of the pathogen [20]. During early infection, Peter's 4-Day suppressive test was used to evaluate schizontocidal activity while the repository test was used to study the prophylactic activity. Rane's test was used to study curative ability during established infection. In all methods, determination of percent inhibition of parasitaemia was the most reliable parameter. A mean parasitaemia level that is $\leq 90 \%$ of that of the vehicle treated animals usually indicates that the test compound is active [21]. In the 4-day

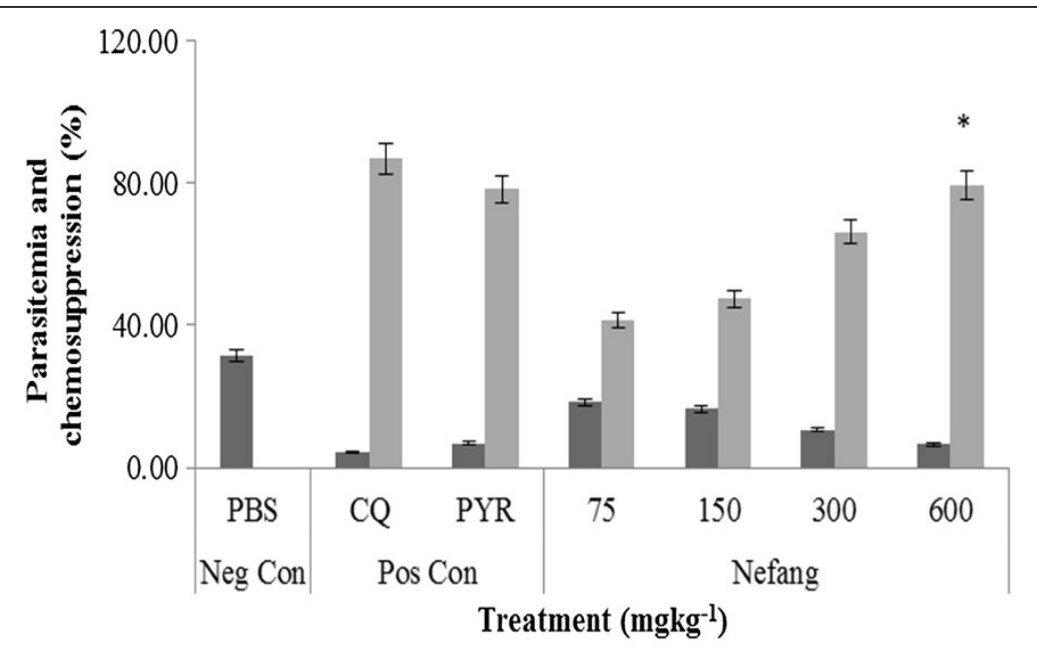

- Parasitemia $₫$ Suppression

Figure 5 Prophylactic activity of Nefang against early P. berghei infection in BALB/c mice. *Compared to negative control (Neg Con); \#Compared to positive control (Pos Con); Significant difference: $p<0.05$. CQ-chloroquine; PYR-pyrimethamine. 


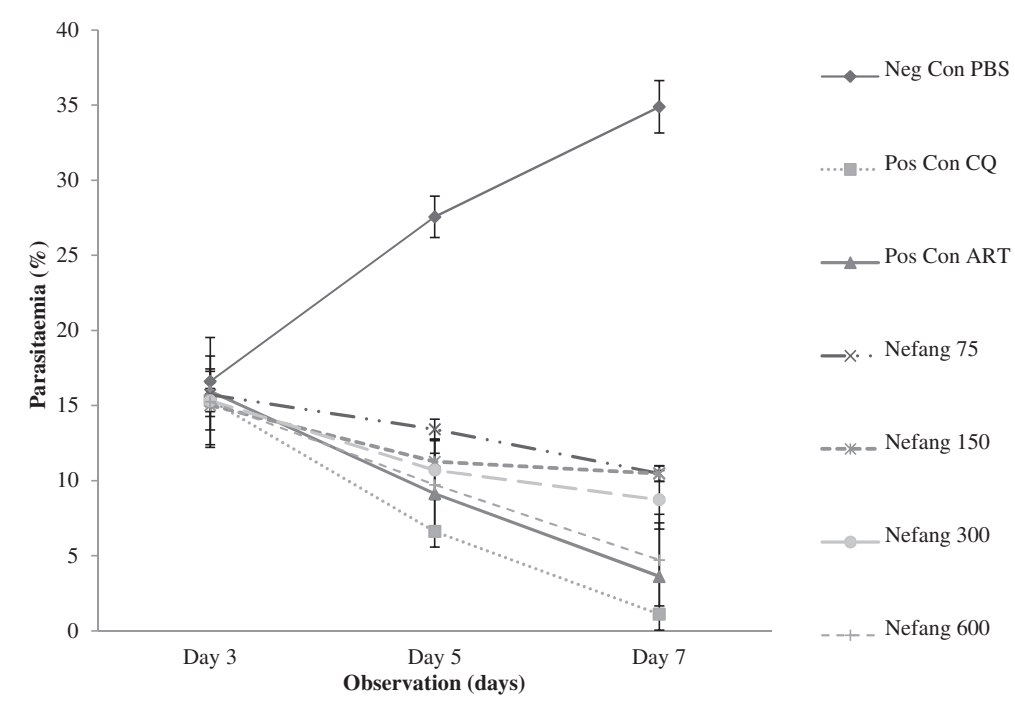

Figure 6 Curative effect of Nefang aqueous extract against established $P$. berghei infection in Wistar rats. Each data point represents the Mean \pm SD for each group of rats, $\mathbf{n}=\mathbf{3}$. Neg Con-negative control; Pos Con-positive control; CQ-chloroquine; ART-artesunate.

suppressive activity, Nefang and $M i B / P g$ significantly reduced parasitaemia (in both Plasmodium spp) in animal models in a dose-dependent manner, with Nefang exhibiting anti-malarial activities comparable to that of the standard drugs tested. The repository test revealed the same dose-dependent chemosuppression by Nefang. In the curative activity, the dose-dependent activity of Nefang at the highest experimental dose was observed from Day 2 of treatment. Though its activity was lower than that of $\mathrm{CQ}$, it was comparable to that of ART. Furthermore, we observed that the survival time of Nefang-treated animals was prolonged in a dose-dependent manner (Figure 7). Nefang caused a dose-dependent reduction of pyrexia and loss of body weight in infected animals. Body weight loss and temperature reduction are hallmarks of malaria infection in animal models [22], suggesting that an effective plantderived anti-malarial agent should prevent body weight loss in Plasmodium infected animals. This dose-dependent preventive activity indicates that Nefang does not have any adverse effect in experimental animals at the doses tested and as observed in our oral acute toxicity studies.

In acute toxicity testing, doses higher than $5,000 \mathrm{mgkg}^{-1}$ bwt are generally not considered as dose related, which is in accordance with the Organization for Economic Corporation and Development (OECD) Guidance Document for Acute Oral Toxicity Testing [23,15]. Compounds with $\mathrm{LD}_{50}$ values lower than $2,000 \mathrm{mgkg}^{-1}$ bwt are generally considered to be relatively safe, because values above this are non-classified. This signifies that Nefang and its constituent aqueous and ethanol extracts can be considered as non-toxic at acute oral administration since the extracts were well tolerated and no observed adverse effect levels were $>5,000 \mathrm{mgkg}^{-1}$. These results are consistent with earlier reports on the cytotoxicity of Nefang [12].

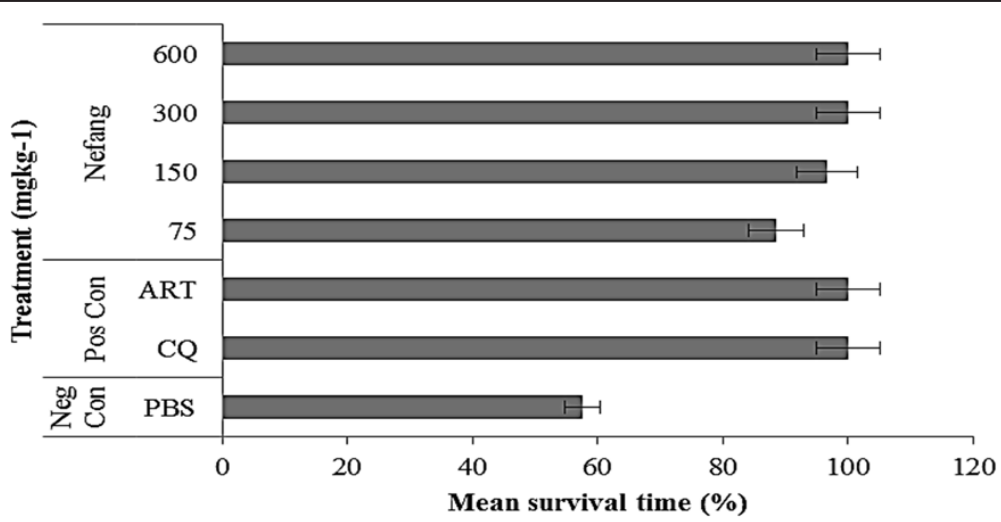

Figure 7 Mean survival time of Wistar rats treated with Nefang aqueous extract during established $P$. berghei infection. Each bar represents the Mean \pm SD for each group of rats, $\mathbf{n}=\mathbf{3}$. Neg Con-negative control; Pos Con-positive control; CQ-chloroquine; ART-artesunate. 


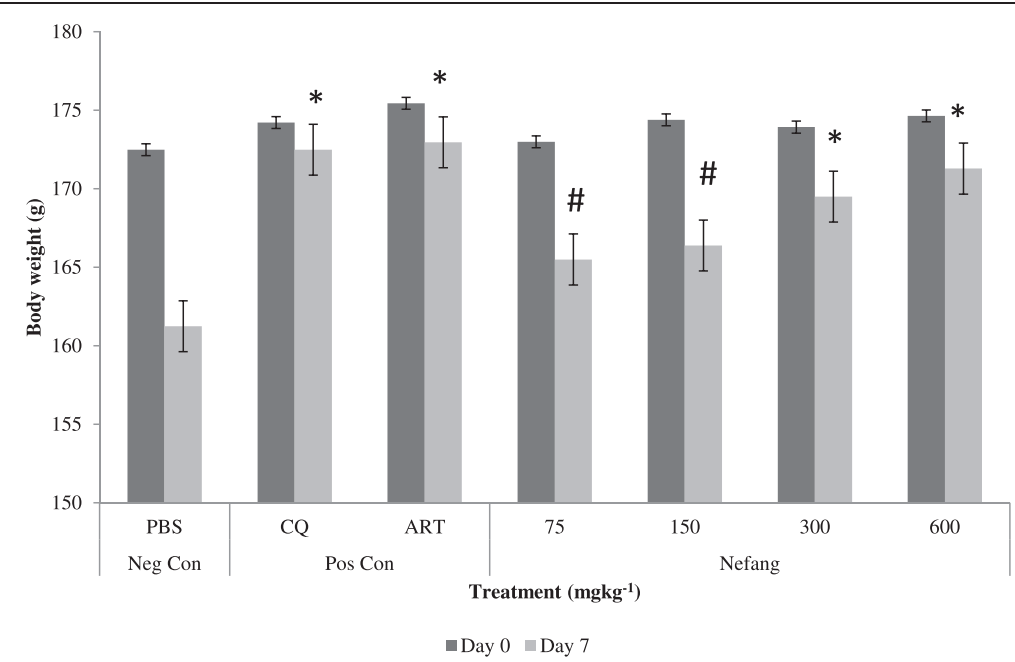

Figure 8 Effect of Nefang aqueous extract on the body weight of $P$. berghei infected rats during established infection (Rane's

Test). *Compared to negative control (Neg Con); \#Compared to positive control (Pos Con); Significant difference: $p<0.05)$. CQ-chloroquine; ART-artesunate.

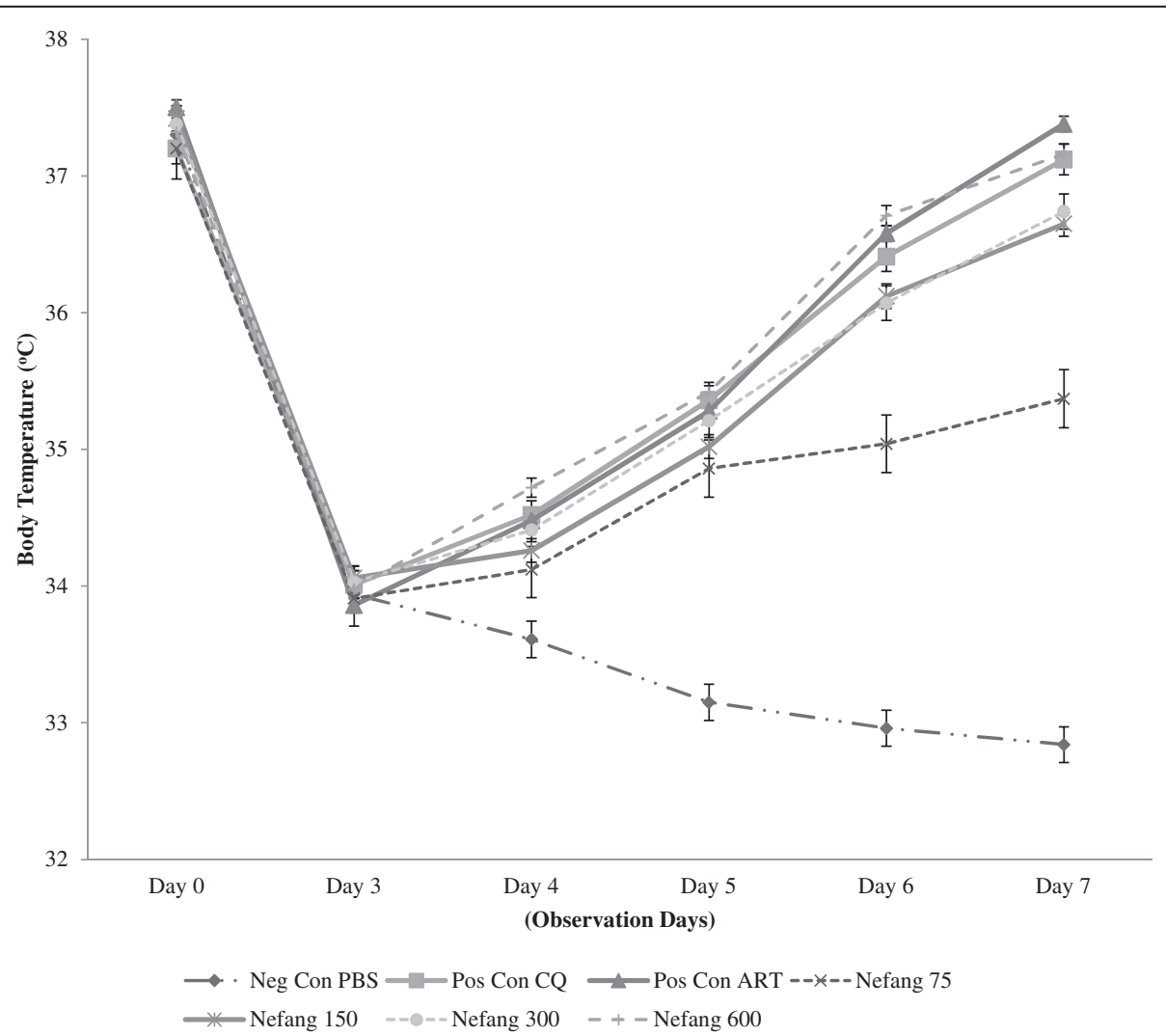

Figure 9 Effect of Nefang aqueous extract on the body temperature of $P$. berghei infected rats during established infection

(Rane's Test). Each data point represents the Mean \pm SD for each group of rats, $n=3$. Neg Con-negative control; Pos Con-positive control; CQ-chloroquine; ART-artesunate. 
Unlike in humans, increase in parasitaemia levels in rodent models usually results in decreased metabolic rates and a consequent decrease in body temperatures [24], which might result in death. An ideal anti-malarial agent would, therefore, prevent this occurrence, an effect observed in Nefang-treated animals. Taken together, these results confirm that Nefang has therapeutic activity against established infection and further confirm the in vitro antiplasmodial activities reported earlier. At the highest dose $\left(600 \mathrm{mgkg}^{-1}\right)$ tested, Nefang exhibited an in vivo suppression of parasitaemia of $>80 \%$, prophylactic activity of $79.5 \%$ and chemotherapeutic effects of $60-80 \%$. In vivo antiplasmodial activity can be classified as moderate, good, and very good if an extract displayed percentage parasitaemia suppression equal to or greater than $50 \%$ at a dose of 500 , 250 and $100 \mathrm{mgkg}^{-1}$ per day, respectively [25], suggesting, therefore, that Nefang has very good activity. This also suggests that the overall anti-malarial activity of the synergistic and additive components identified during the interaction studies [12], over-shadowed the antagonistic interactions. Synergy between different constituents of extracts has been documented for anti-malarial and other pharmacological activities [26,27].

Earlier phytochemical screening of the constituent plant extracts of Nefang showed the presence of alkaloids, anthocyanins, flavonoids, phenols, saponins, tannins, triterpenes and sterols [12], suggesting that the anti-malarial activity of Nefang cannot be attributed only to the active antiplasmodial compounds in the constituent plant extracts. In our earlier in vitro antiplasmodial studies on the constituent plant extracts of Nefang, only M. indica (bark and leaf) and P. guajava showed good activities while the rest showed very weak activities or inactivity. However, there have been reports of good in vivo antiplasmodial and anti-malarial activities exhibited by C. papaya [28], C. citratus [29] and O. gratissimum [30], which are constituent plants of Nefang. As earlier reported, many anti-malarial herbal remedies may exert their anti-infective effects not only by directly affecting the pathogen, but also by indirectly stimulating natural and adaptive defense mechanisms of the host by other mechanisms. Therefore, extracts that can stimulate innate and/or adaptive immunity may be able to contribute to prophylaxis and treatment not only for malaria but for other diseases as well $[31,32]$. This suggests that a combination of the biological activities of the constituent plant extracts of Nefang results in an enhanced overall anti-malarial activity of Nefang. Therefore, an understanding of the underlying pharmacodynamic or pharmacokinetic mechanisms of this action would be very important towards understanding the anti-malarial potentials of Nefang.

\section{Conclusion}

This study provides evidence that Nefang is safe and possesses good in vivo suppressive, prophylactic and curative activities against Plasmodium species. These findings uphold earlier in vitro antiplasmodial activities and confirm the synergistic interactions between its constituents plant extracts. It further suggests that the observed in vivo anti-malarial activity of Nefang may result from the synergistic biological activities of its constituent plant extracts. Hence, Nefang represents a promising source of anti-malarial agents for downstream clinical development.

\section{Additional files}

Additional file 1: Body temperature (Day 0 and Day 4) of
Plasmodium infected animals treated with aqueous extract of
Nefang and its active components in the 4-day suppressive test.
Additional file 2: Body weight (Day 0 and Day 4) of Plasmodium
infected animals treated with aqueous extract of Nefang and its
active components in the 4-day suppressive test.
Additional file 3: Effect of Nefang aqueous extract on the body
weight of $P$. berghei infected rats during established infection
(Rane's Test).
Additional file 4: Effect of Nefang aqueous extract on the body
temperature of $P$. berghei infected rats during established infection
(Rane's Test).

Competing interests

The authors declare that they have no competing interests.

\section{Authors' contribution}

PAT conceived the study, carried out in vivo toxicity/anti-malarial studies and drafted the manuscript; FAO participated in the design and execution of the in vivo anti-malarial studies; LSA participated in the design and execution of the in vivo antiplasmodial (suppressive activity) studies; GAA participated in the design and execution of the in vivo toxicity studies; ANG participated in the conception, design and general supervision of the studies. All authors read and approved the final draft of the manuscript.

\section{Acknowledgements}

The authors are thankful to Institut Pasteur Korea for hosting PAT, and for providing both material and reagent support during the study. We also acknowledge the material and technical support of Dr. Ozwara HS, Institute of Primate Research (IPR), Nairobi, Kenya; Mr. Mbithi E and Ms. Muthini F, Department of Medical Microbiology; Dr. Abuga KO and Mr. Mugo HN, Department of Pharmaceutical Chemistry, University of Nairobi, Kenya.

\section{Author details}

${ }^{1}$ Centre for Research on Medicinal Plants and Traditional Medicine, Institute of Medical Research and Medicinal Plants Studies (IMPM), P. O. Box 6163, Yaoundé, Cameroon. 'Department of Pharmacology and Pharmacognosy, University of Nairobi, P. O. Box 19676-00202, Nairobi, Kenya. ${ }^{3}$ Early Drug Discovery Programme, Institut Pasteur Korea, Sampyeong-dong 696, Bundang-gu, Seongnam-si, Gyeonggi-do, South Korea. ${ }^{4}$ Current address: Public Health and Epidemiology Unit, Centre Pasteur du Cameroun, P. O. Box 1274 Yaoundé, Cameroon.

Received: 6 August 2014 Accepted: 15 November 2014 Published: 25 November 2014

\section{References}

1. WHO: World Malaria Report 2013. World Health Organization. Geneva: http://www.who.int/malaria/publications/world_malaria_report_2013/en/.

2. Oliveira AB, Dolabela MF, Braga FC, Jacome RL, Varotti FP, Povoa MM: Plant-derived antimalarial agents: new leads and efficient phythomedicines. Part I Alkaloids Acad Bras Cienc 2009, 81:715-740.

3. Castellanos JRG, Prieto JM, Heinrich M: Red Lapacho (Tabebuia impetiginosa)- a global ethnopharmacological commodity? J Ethnopharmacol 2009, 121:1-13. 
4. Kong DX, Li XJ, Zhang HY: Where is the hope for drug discovery? Let history tell the future. Drug Discov Today 2009, 14:115-119.

5. Wagner H, Ulrich-Merzenich G: Synergy research: approaching a new generation of phytopharmaceuticals. Phytomedicine 2009, 16:97-110.

6. Ginsburg H, Deharo E: A call for using natural compounds in the development of new antimalarial treatments - an introduction. Malar 」 2011, 10(Suppl 1):S1.

7. Ganesan A: The impact of natural products upon modern drug discovery. Curr Opin Chem Biol 2008, 12:306-317.

8. Ma XH, Zheng CJ, Han LY, Xie B, Jia J, Cao ZW, Li YX, Chen YZ: Synergistic therapeutic actions of herbal ingredients and their mechanisms from molecular interaction and network perspectives. Drug Discov Today 2009, 14:579-588.

9. Nosten F, White NJ: Artemisinin-based combination treatment of falciparum malaria. Am J Trop Med Hyg 2007, 77:181-192.

10. Tarkang PA, Okalebo FA, Agbor GA, Tsabang N, Guantai AN, Rukunga GM: Indigenous Knowledge and folk use of a polyherbal antimalarial by the Bayang Community, South West Region of Cameroon. J Nat Prod Plant Res 2012, 2:372-380.

11. Tarkang PA, Atchan APN, Kuiate J, Okalebo FA, Guantai AN, Agbor GA: Antioxidant potential of a polyherbal antimalarial as an indicator of its therapeutic value. Adv Pharmacol Sci 2013, 2013:678458.

12. Tarkang PA, Franzoi KD, Lee $S$, Lee E, Vivarelli $D$, Freitas-Junior L, Liuzzi M, Tsabang N, Ayong LS, Agbor GA, Okalebo FA, Guantai AN: In vitro antiplasmodial activities and synergistic combinations of differential solvent extracts of the polyherbal product. Nefang BioMed Res Int 2014, 2014:835013

13. Willcox M: Improved traditional phytomedicines in current use for the clinical treatment of malaria. Planta Med 2011, 77:662-671.

14. Handa SS, Khanuja SPS, Longo G, Rakesh DD: Extraction Technologies for Medicinal and Aromatic Plants. Trieste, Italy: ICS-UNIDO International Centre for Science and High Technology; 2008:266.

15. Organization for Economic Cooperation Development: Guidance Document on Oral Toxicity Testing 423 and 425, Environment, Series on testing and Assessment N²4. Paris, France: Health and Safety Publications; 2001:24.

16. Basir R, Fazalul Rahiman SS, Hasballah K, Chong WC, Talib H, Yam MF, Jabbarzare M, Tie TH, Othman F, Moklas MAM, Abdullah WO, Ahmad Z: Plasmodium berghei ANKA infection in ICR mice as a model of cerebral malaria. Iranian J Parasitol 2012, 7:62-74.

17. Knight DJ, Peters W: The antimalarial action of $\mathrm{N}$-benzyloxy dihydrotriazines. The action of cycloguanil (BRL50216) against rodent malaria and studies on its mode of action. Ann Trop Med Parasitol 1980, 74:393-404.

18. Peters W: Drug resistance in Plasmodium berghei. Vincke and Lips, 1948; 1. Chloroquine resistance. Exp Parasitol 1965, 17:80-89.

19. Ryley JF, Peters W: The antimalarial activity of some quinone esters. Ann Trop Med Parasitol 1970, 84:209-222.

20. Waako PJ, Gumede B, Smith P, Folb PI: The in vitro and in vivo antimalarial activity of Cardiospermum halicacabum and Momordica foetida. J Ethnopharmacol 2005, 99:137-143.

21. Peter IT, Anatoli VK: The Current Global Malaria Situation. Malaria Parasite Biology, Pathogenesis, and Protection. Washington DC, USA: ASM Press; 1998:11-22.

22. Langhorne J, Quin SJ, Sanni LA: Mouse models of blood-stage malaria infections: immune responses and cytokines involved in protection and pathology. In Malaria Immunology. 2nd edition. Edited by Perlmann P, Troye Blomberg M. Stockholm: Karger; 2002:204-228.

23. Hayes AW: Guidelines of Acute Oral Toxicity Testing. 2nd edition. New York: Raven Press Ltd; 1987:185.

24. Chinchilla M, Guerrero OM, Abarca G, Barrios M, Castro O: An in vivo model to study the anti-malaria capacity of plant extracts. Rev Biol Trop 1998, 46:1-7.

25. Deharo E, Bourdy G, Quenevo C, Munoz V, Ruiz G, Sauvain M: A search for national bioactive compounds in Bolivia through a multidisciplinary approach. Part V. Evaluation of the antimalarial activity of plants used by the Tecana Indians. J Ethnopharmacol 2001, 77:91-98.

26. Williamson EM: Synergy and other interactions in phytomedicines. Phytomedicine 2001, 8:401-409.

27. Houghton PJ: Synergy and polyvalence: paradigms to explain the activity of herbal products. In Evaluation of Herbal Medicinal Products. Edited by Houghton PJ, Mukherjee PK. London: Pharmaceutical Press; 2009:85-94.
28. Ofori-Attah K, Oseni LA, Quasie O, Antwi S, Tandoh M: A comparative evaluation of in vivo antiplasmodial activity of aqueous leaf exracts of Carica papaya, Azadirachta indica, Magnifera indica and the combination thereof using Plasmodium infected BALB/c mice. Int J Applied Biol Pharm Tech 2012, 3:372-378.

29. Melariri $P$, Campbell W, Etusim $P$, Smith $P$ : In vitro and in vivo antiplasmodial activities of extracts of Cymbopogon citratus Staph and Vernonia amygdalina Delile leaves. J Nat Prod 2011, 4:164-172.

30. Murithi CK, Fidahusein DS, Nguta JM, Lukhoba CW: Antimalarial activity and in vivo toxicity of selected medicinal plants naturalised in Kenya. Int J Edu Res 2014, 2:395-406.

31. Masihi KN: Immunomodulatory agents for prophylaxis and therapy of infections. Int J Antimicrob Agents 2000, 14:181-191.

32. Muniz-Junqueira MI: Immunomodulatory therapy associated to antiparasite drugs as a way to prevent severe forms of malaria. Curr Clin Pharmacol 2007, 2:59-73.

\section{doi:10.1186/1475-2875-13-456}

Cite this article as: Arrey Tarkang et al:: Anti-malarial activity of a polyherbal product (Nefang) during early and established Plasmodium infection in rodent models. Malaria Journal 2014 13:456.

\section{Submit your next manuscript to BioMed Central and take full advantage of:}

- Convenient online submission

- Thorough peer review

- No space constraints or color figure charges

- Immediate publication on acceptance

- Inclusion in PubMed, CAS, Scopus and Google Scholar

- Research which is freely available for redistribution
( Biomed Central 